\title{
Numerical Simulation on the specimen dynamic plastic deformation behaviour in the torsional split Hopkinson bar test
}

\author{
Chen Gang ${ }^{1,2, *}$, Huang Xicheng ${ }^{1,2}$, Chen Junhong ${ }^{1,2}$, and Zhong Weizhou ${ }^{1,2}$ \\ ${ }^{1}$ Institute of systems engineering, China Academy of Engineering Physics, Mianyang, 621999, China \\ ${ }^{2}$ Shock and Vibration of Engineering Materials and Structures Key Laboratory of Sichuan Province, Mianyang Sichuan 621999, \\ China
}

\begin{abstract}
The torsional split Hopkinson bar (SHB) is an important method to study the dynamic shear behaviour and shear localization of materials under high strain rates. Different specimen sizes were used in literatures, and the size of the specimen might have an effect on the experimental results. Numerical simulation on torsional SHB tests was carried out with LS-DYNA. The strain signal on the incident and transmitted bars were obtained from the simulation just as the experiment. Then the numerical strain-stress relationship of the material was derived from the numerical strain signal using the experiments data process of torsional SHB. The agreement between numerically derived strain-stress results and the specimen material properties specified in numerical modelling indicates that the torsional SHB is applicable to study the dynamic shear behaviour of materials under high strain rates. The specimen gauge diameter has no significant effect on the dynamic torsional test result. However, higher adhesive strength is required to fix the larger gauge diameter specimen on the bars. The specimen gauge thickness has little effect on the experimental results with a modified formula to calculate the specimen stress. Still, the increase of specimen gauge thickness will lead to the increase of non-uniformity of specimen stress and strain (strain rate). Based on the simulation analysis, suggestions on the specimen size design are given as well.
\end{abstract}

\section{Introduction}

The torsional split Hopkinson bar (SHB) technology is an important method to study the dynamic shear behaviour and shear localization of materials under high strain rates. In this technique, a short specimen (thinwalled tube with two flanges) fixed between two elastic bars undergoes a high strain rate shear plastic deformation by a torsional stress wave loads generated in the incident bar. Torsional SHB technique can effectively avoid the geometric dispersion of wave propagation, the end face friction, radial inertia and the geometric in-homogeneity in large strain.

The torsional SHB was proposed by Baker and Yew in 1966 [1]. This method was studied and improved by many researchers [2-5]. A large number of materials have been studied on the high strain rate shear mechanical properties and adiabatic shear with this experimental method [6-12]. Various sizes of specimen gauge length, diameter and thickness were chosen in different researches. In this paper, the numerical simulation of torsion SHB loading process is carried out by LS-DYNA. The strain signal on the incident and transmitted bars were obtained from the simulation just as the experiment. Then the numerical strain-stress relationship of the material was derived from the numerical strain signal using the experiments data process of torsional SHB. The effects of specimen size are discussed based on the numerical experiment results.

The distribution of stress and plastic deformation of the specimen in the loading process are analysed further. A series of numerical experiments are carried out by changing the size of the specimen, the influence of the parameters on the experimental results is analysed, and suggestions on the specimen size design are given.

\section{Finite element model}

In the torsional SHB experiment, two long elastic cylindrical bars are coaxial fixed with the specimen between them. A torsional stress wave is generated in the incident bar by releasing an initial stored torque between the loading wheel and a clamp. When the wave translated to the interface between the incident bar and the specimen, a part of it is reflected, while another part goes through the specimen and develops the transmitted wave in the output bar. The specimen gauge section undergoes high strain rate deformation during the wave translating process. Based on one-dimensional stress wave theory, the load and displacement process of the specimen can be obtained by the strain history measurement in the long bars. With the assumption of uniform deformation in specimen (neglecting the

* Corresponding author:chengang@caep.cn 
propagation of the stress wave in the specimen), the dynamic shear stress, strain and strain rate of the specimen are calculated then.

The numerical simulation of torsional SHB was conducted with LS-DYNA. The input bar, output bar and specimen are included in the simulation, as shown in figure 1 . The dimensions of both bars are diameter of 25 $\mathrm{mm}$ and length of $2000 \mathrm{~mm}$. The inner diameter of the specimen gauge section is $10 \mathrm{~mm}$, and the thickness is 1 $\mathrm{mm}$. The outer diameter of the specimen flange is 25 $\mathrm{mm}$. The mesh dimension of bars is $2 \mathrm{~mm}$. In order to effective describe the in-homogeneous deformation in radial direction, the thin-walled gauge length section of the specimen is meshed with five layers along thickness, and the specimens are divided into 120 elements in circumference.

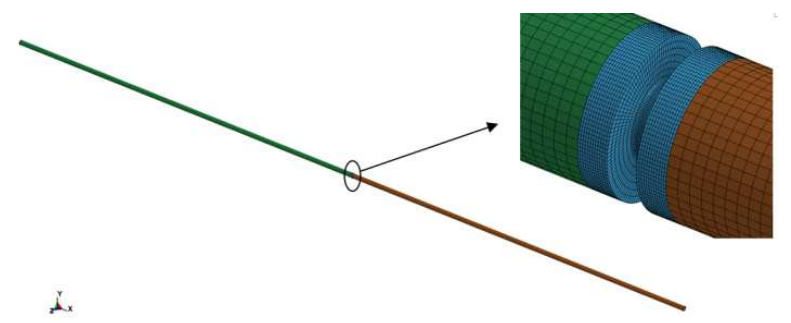

a) Entire experiment model

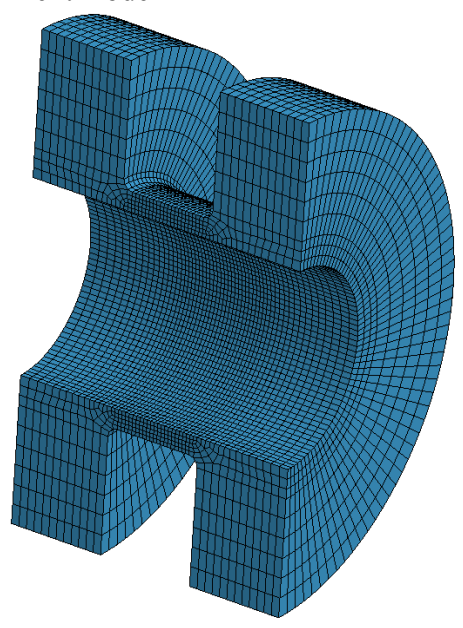

b) Cross section of specimen model

Fig. 1. The FEM model of torsional SHB in the simulation.

In the numerical simulation, a time dependent angular velocity boundary condition is applied at the front end of the input bar to simulate loading process in the experiment.

The specimen and the bars are bonded by adhesive in the experiment. This connection is simulated with the function CONTACT TIED SURFACE TO SURFACE in LS-DYNA. The numerical incident and transmitted wave in the bars are shown in figure 2 in the case of the two long bars fixed together with this function. It can be seen that these two waves are exactly the same. This indicates that the connection simulation of TIE method has no effect on the propagation of the stress wave, and the propagation of shear wave in the bars can be described effectively with the model.
The elastic constitutive model (*MAT_ELASTIC) is applied for the bars, while the elastic-plastic model (*MAT_PLASTIC_KINEMATIC) for the specimen.

The constitutive model parameters of the bars and specimen are shown in Table 1 . In the numerical simulation, the strain rate effect of specimen material property is not taken into account so that it is convenient for the comparison of the results obtained by numerical experiment with the input parameters.

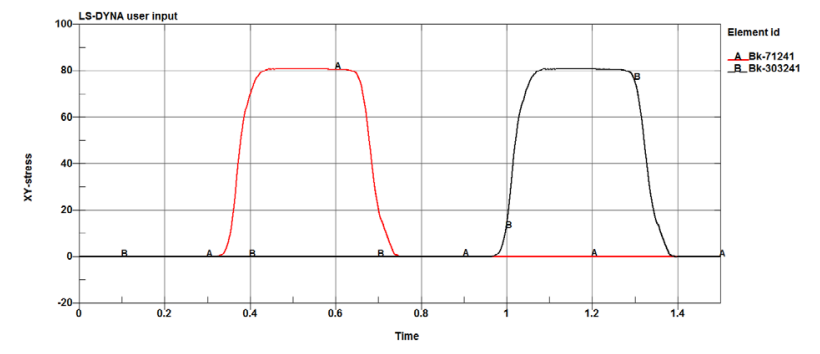

Fig. 2. The incident and transmitted stress wave curve of two bars fixed together.

Table 1. Constitutive model parameters of bars and the specimen.

\begin{tabular}{|c|c|c|c|c|c|}
\cline { 2 - 6 } \multicolumn{1}{c|}{} & $\begin{array}{c}\text { Density } \\
\mathrm{kg} / \mathrm{m}^{3}\end{array}$ & $\begin{array}{c}\text { Elastic } \\
\text { modulus } \\
\mathrm{GPa}\end{array}$ & $\begin{array}{c}\text { Poisson's } \\
\text { ratio }\end{array}$ & $\begin{array}{c}\text { Yield } \\
\text { stress } \\
\mathrm{MPa}\end{array}$ & $\begin{array}{c}\text { Hardening } \\
\text { modulus } \\
\mathrm{MPa}\end{array}$ \\
\hline bars & 4400 & 110 & 0.28 & - & - \\
\hline specimen & 7800 & 200 & 0.28 & 400 & 500 \\
\hline
\end{tabular}

\section{Simulation results and analysis}

\subsection{Results of numerical experiments}

The output strain information of the element in the numerical simulation contains the six components in Cartesian coordinates. The shear strain signal of bar surface at middle section is obtained through coordinate transformation. To obtain the strain signal just as the experiment, the distance between the element centre and the bar surface is taken into account in the transformation. The final curve in the numerical experiment is shown in figure 3 . The shear stress-strain curve of the specimen material is obtained based on the curve in figure 3 and is shown in figure 4.

The material parameters are inputted as effective stress and strain in the numerical simulation. The material property result of the torsional SHB experiment is described as shear stress-strain relations. The shear stress-strain curve needs to be converted to the same formation of the input. According to the theory of elastic-plastic mechanics, the effective stress is expressed as:

$$
\sigma_{g f f}=\frac{1}{\sqrt{2}} \sqrt{\left(\sigma_{1}-\sigma_{2}\right)^{2}+\left(\sigma_{2}-\sigma_{3}\right)^{2}+\left(\sigma_{3}-\sigma_{1}\right)^{2}}
$$


In the stress state of pure shear, $\quad \sigma_{1}=-\sigma_{3}=\tau$,

$\sigma_{2}=0$, it can be obtained that $\sigma_{\text {eff }}=\sqrt{3} \tau$.

The effective strain is expressed as

$$
\varepsilon_{\varepsilon f f}=\frac{\sqrt{2}}{3} \sqrt{\left(\varepsilon_{1}-\varepsilon_{2}\right)^{2}+\left(\varepsilon_{2}-\varepsilon_{3}\right)^{2}+\left(\varepsilon_{3}-\varepsilon_{1}\right)^{2}}
$$

and in the stress state of pure shear $\quad \varepsilon_{1}=-\varepsilon_{3}=\gamma / 2, \quad \varepsilon_{2}=0$, it can be obtained that $\quad \varepsilon_{\text {eff }}=\frac{2}{\sqrt{3}} \varepsilon_{1}=\gamma / \sqrt{3}$ 。

The result of the numerical experiment is compared with the inputted parameter, as shown in Fig. 5. The difference between them at the yield point is about 0.1 percent, and the maximum difference is less than 2 percent.

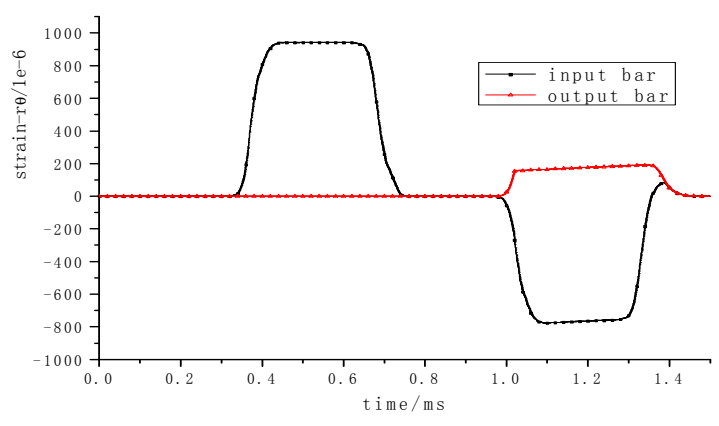

Fig.3. Shear strain curves at the middle of the input bar and output bar.

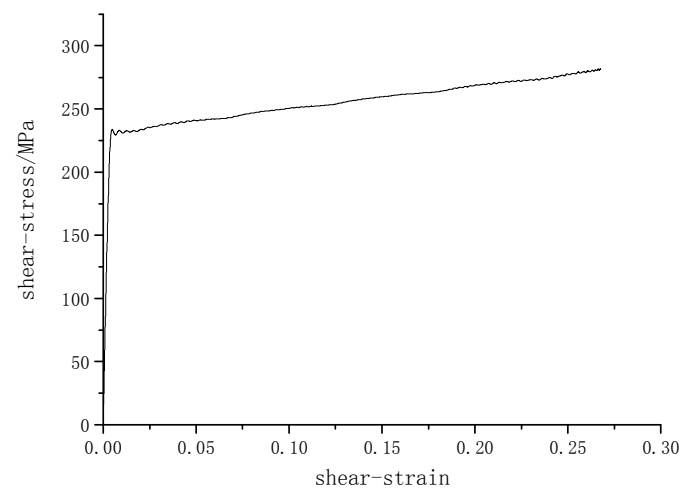

Fig.4. Result of numerical experiment.

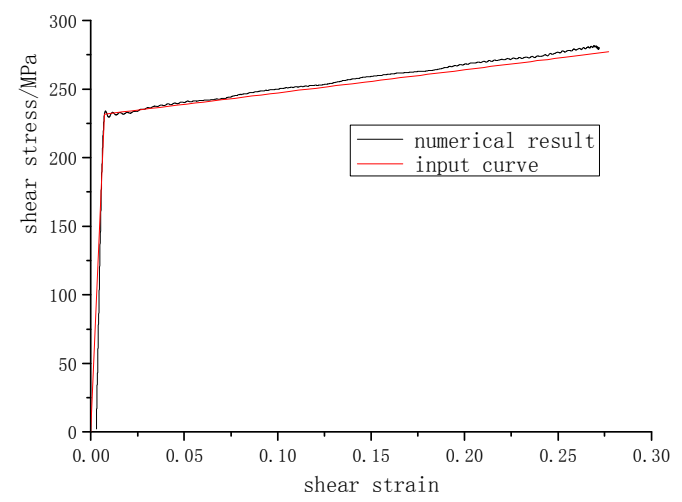

Fig.5. Comparison of the numerical experiment result and the imputed parameters.

\subsection{Strain and stress uniformity in the specimen}

Contours of effective plastic strain and stress of the specimen in the loading process (at the later stage) are given in figure 6 . The effective stress is uniform in the whole gauge section of specimen, while the plastic strain near the flange and chamfer is smaller than that in the middle part. To identify the deformation uniformity of the specimen quantitatively, the three elements in the middle of the specimen at the outer layer, the middle layer and the inner layer respectively are chosen as shown in figure 7 . The effective stress and plastic strain histories of these elements are given in figure 7 . The maximum effective stress difference between the outer layer and the inner layer element in the loading process is about 2.6 percent, and the difference of effective plastic strain is 15.9 percent. The strain of the cylinder material is proportional to the distance from the axis. The inner diameter of the specimen gauge section is 10 $\mathrm{mm}$, and the outer diameter is $12 \mathrm{~mm}$. As a result, the strain difference between the outer surface and the inner surface of the specimen gauge section is $20 \%$. In the numerical simulation, the element has a certain scale, and the simulation result gives the value at the element centre. The theoretic results are consistent with the numerical simulation results according to the central coordinate of the element.
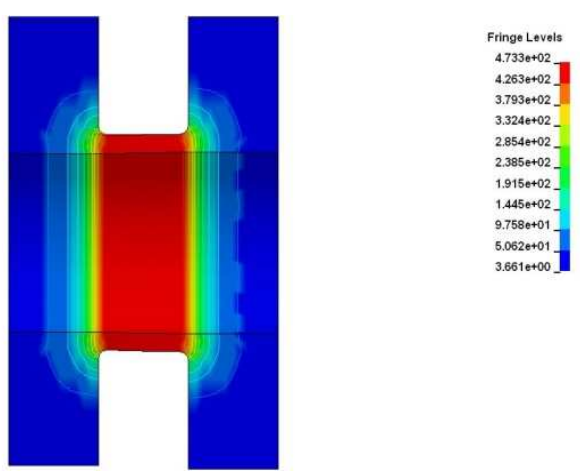

a) effective stress
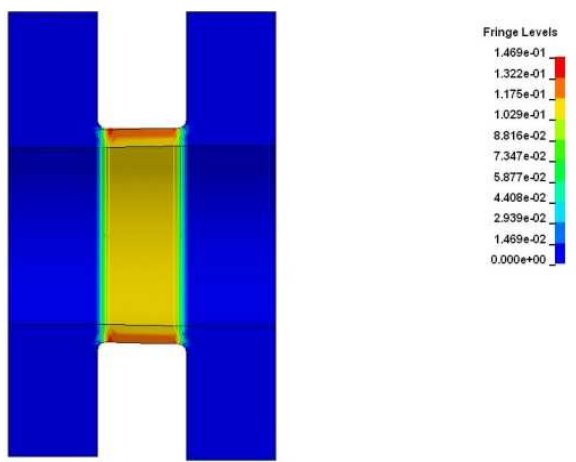

b) effective plastic strain

Fig.6. Contours of the specimen deformation in the loading process. 


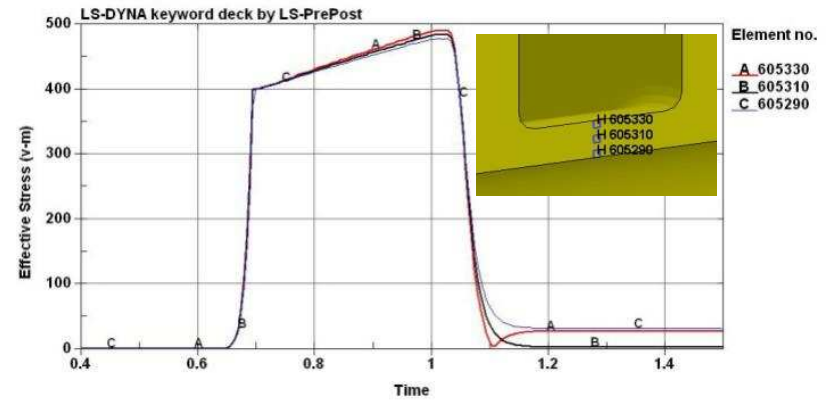

a) effective stress

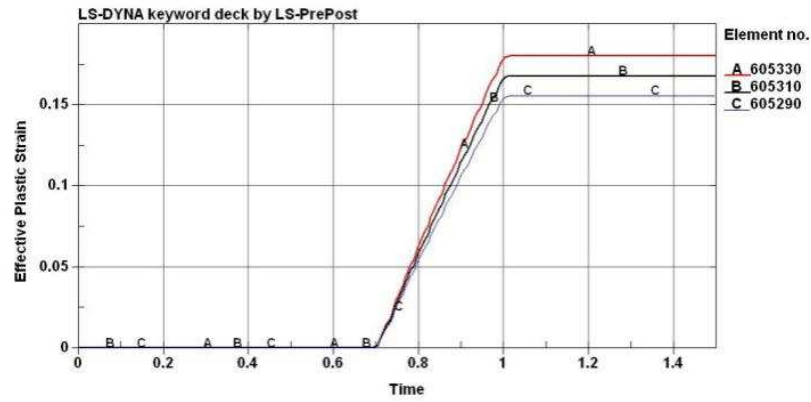

b) effective plastic strain

Fig. 7. Comparison of specimen material response during the loading process.

\subsection{Effects of specimen gauge thickness}

The numerical simulations of torsional SHB loading process are carried out with four specimen gauge thicknesses of $0.5 \mathrm{~mm}, 1 \mathrm{~mm}, 2 \mathrm{~mm}$ and $4 \mathrm{~mm}$ respectively, under the condition of constant inner diameter with $10 \mathrm{~mm}$. The specimen model is shown in figure 8 , and they have no chamfer. The corresponding curves of numerical experiments are shown in figure 9. With the increase of specimen thickness, the amplitude of corresponding transmission wave signal also increases. When the amplitude of input signal is not change, the corresponding strain rate (proportional to the reflected wave) would decrease as the specimen gauge thickness increases. In order to keep the strain rate to be constant, the amplitude of incident pulse should be increased.

To obtain the shear stress time relationship of the specimen material, the torque applied to the specimen need to be calculated. Because the specimen is fixed to the bar end, the torque and angular displacement of the specimen are the same as those of the bar end at the specimen-bar interface, and then these curve can be calculated from the curve in figure 9.

The relationship between the torque of the round tube and the shear stress of the tube material is

$$
M=\int_{r_{s}}^{R_{s}} 2 \pi r d r * \tau * r=2 \pi \int_{r_{s}}^{R_{s}} r^{2} \tau d r
$$

where, $\mathrm{M}$ is the torque, $R_{s}$ is the outer radius of the specimen gauge section, $r_{s}$ is the inner radius of the specimen gauge section, $\tau$ is shear stress.
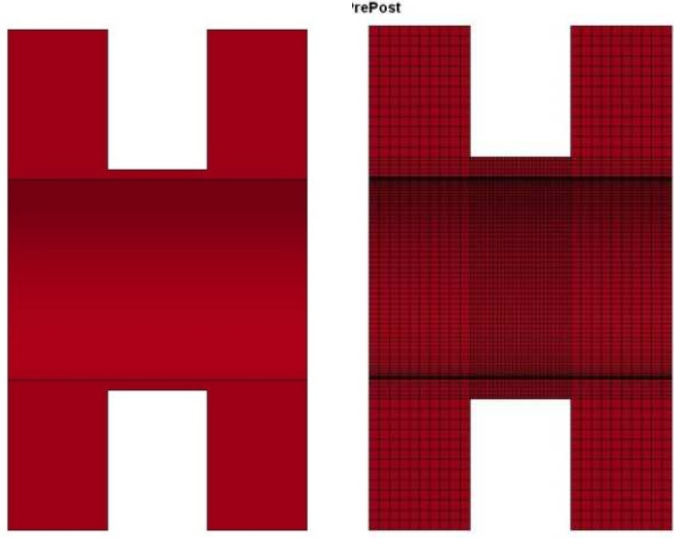

a) $0.5 \mathrm{~mm}$

b) $1 \mathrm{~mm}$
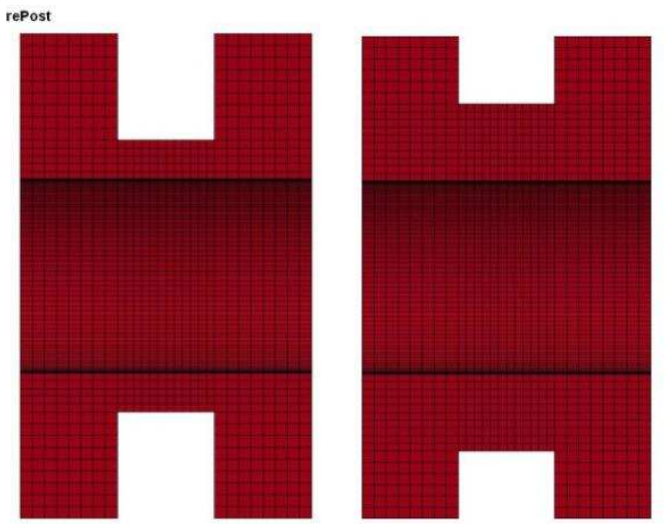

c) $2 \mathrm{~mm}$

d) $4 \mathrm{~mm}$

Fig.8. Cross section of specimen with four gauge thickness.

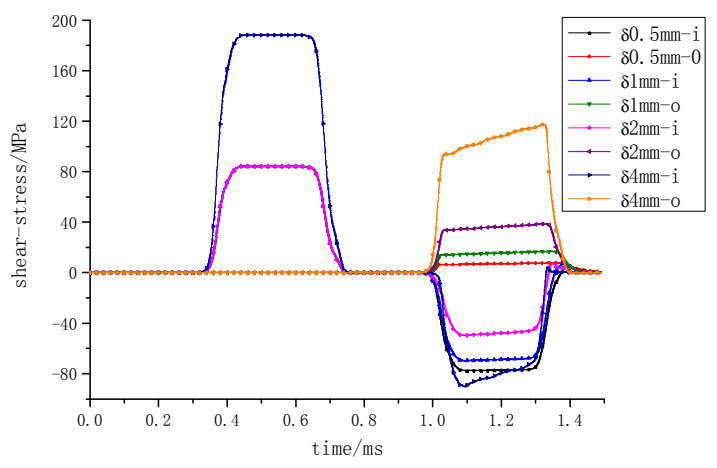

Fig.9. curves of numerical experiments for four gauge thickness specimen.

Under the stress uniformity assumption of thin walled cylinder, formula (1) can be given as

$$
M=2 \pi r \delta * \tau * \bar{r}=\frac{1}{2} \pi\left(R_{s}+r_{s}\right)^{2} \delta \tau
$$


where, $\delta$ is the thickness of the thin-walled specimen gauge section, $\bar{r}$ is the mean radius of the specimen gauge section.

Under the assumption of uniform stress of thin walled cylinder, formula (1) can be directly integrated to obtain more accurate expression as

$$
M=\frac{2}{3} \pi\left(R_{s}^{3}-r_{s}^{3}\right) * \tau=\frac{2}{3} \pi\left(R_{s}^{2}+r_{s}^{2}+R_{s} r_{s}\right) \delta \tau
$$

Under the assumption of material stress is proportional to the radius, the formula (1) can be expressed as

$$
M=\frac{J_{s}}{\bar{r}} \tau=\frac{\pi\left(D_{s}^{4}-d_{s}^{4}\right)}{8\left(D_{s}+d_{s}\right)} \tau=\pi\left(R_{s}^{2}+r_{s}^{2}\right) \delta \tau
$$

Both the formula (2) $[3,5,7,10-13]$ and the formula (4) $[1,2,6]$ have been adapted to analysis the torsional SHB experimental data in literature respectively. The formula (2) is the simplification and approximation of formula (3). The formula (4) contains the assumption that the stress is proportional to the radius, and this assumption is established under complete elastic conditions only. These three formulas are used to further analyse the curves obtained from numerical experiments for specimens with different thin-wall thicknesses.

The comparison of the shear stress-strain curves obtained with three different stress calculation methods under the three wall thicknesses of $1 \mathrm{~mm}, 2 \mathrm{~mm}$ and $4 \mathrm{~mm}$ are given in figure 10. The inputted data is given in figure 10 also. The difference between results of specimen gauge thickness below $1 \mathrm{~mm}$ from these three methods is very small, and they are consistent with the input data. With the increase of specimen gauge thickness, difference between results from the three methods increases, while the result from formula (3) is always consistent with the input data.

\subsection{Effects of specimen gauge diameter}

The numerical simulation was carried out on the two cases of the specimen with inner diameter of $10 \mathrm{~mm}$ and $15 \mathrm{~mm}$, respectively. In the both cases, the thickness of thin-wall gauge section is $1 \mathrm{~mm}$ and length of test gauge is $5 \mathrm{~mm}$. The influence of specimen radius on the experiment was analysed based on the simulation. The "measure" waveform curves of numerical experiments under the two cases are given in figure 11. It can be seen that the increase of the inner diameter of the specimen leads to the corresponding increase of the amplitude of transmitted wave, and the increase of the amplitude is proportional to the increase of the radius. In the simulation, the amplitude of incident wave in the case of inner diameter of $15 \mathrm{~mm}$ is increased to make the amplitude of the reflected wave in both cases be equivalent. The experimental stress-strain curves obtained under the two cases are given in figure 12. It can be seen that the shear stress-strain curves obtained from the specimens with different internal diameters are consistent. Although the amplitudes of transmitted wave in the two conditions are the same, the final strain (or strain rate) in the case of the $15 \mathrm{~mm}$ diameter is much larger than that of $10 \mathrm{~mm}$.

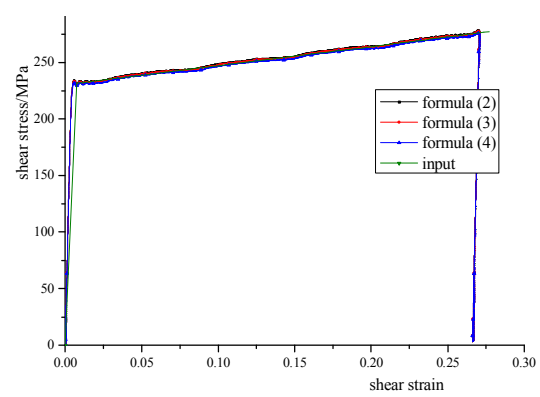

a) specimen gauge thickness of $1 \mathrm{~mm}$

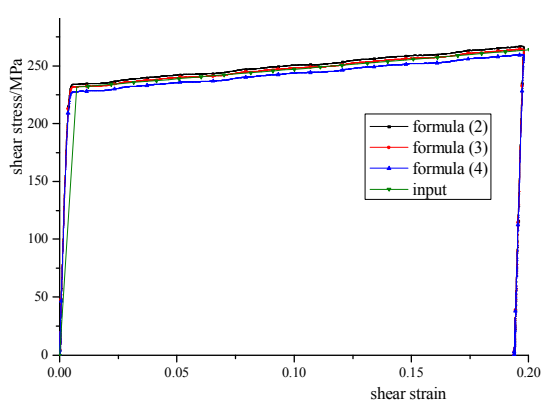

b) specimen gauge thickness of $2 \mathrm{~mm}$

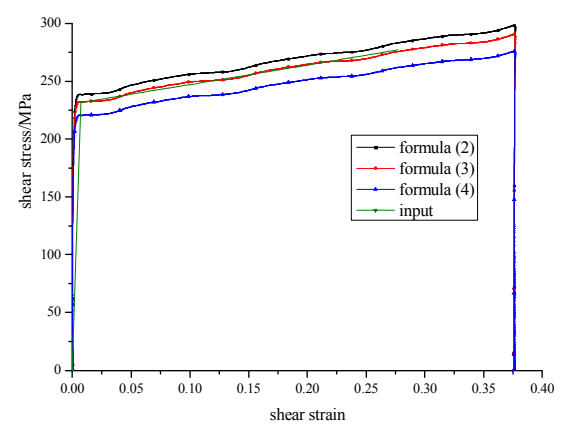

c) specimen gauge thickness of $1 \mathrm{~mm}$

Fig.10. Comparison of specimen response with different gauge thickness from three methods.

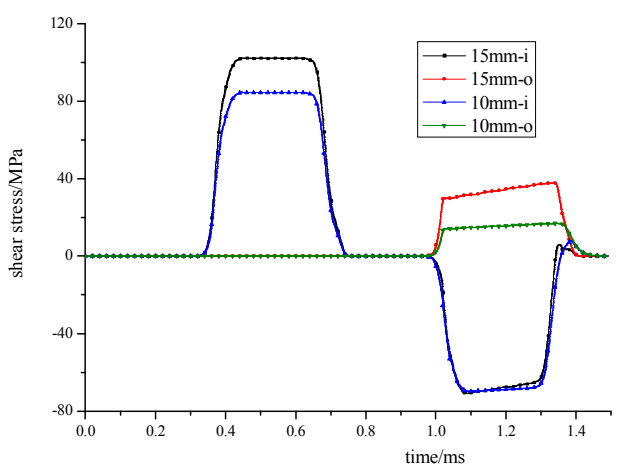

Fig.11. The numerical experiment curves of two inner diameter specimen.

When the thickness keeps constant, the increase of specimen gauge radius will lead to the increase of 
transmitted wave amplitude. Still, the change of specimen gauge radius has little effect on the experimental strain-stress relationship.

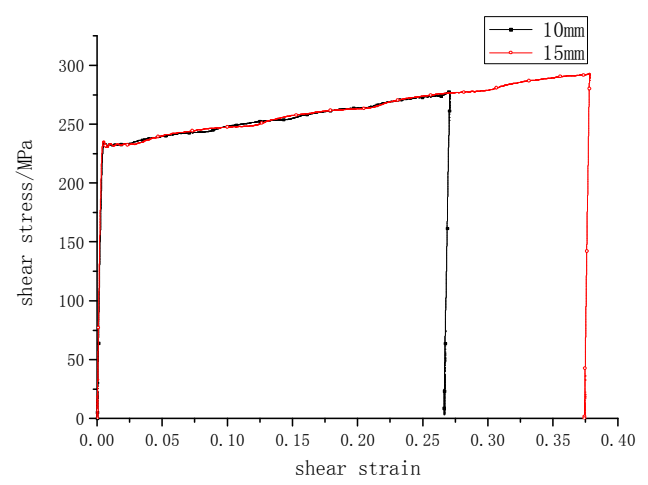

Fig.12. Results of specimen material stress-strain curve in two cases.

If the specimen is fixed to the input bar and output bar with high strength epoxy, the variation of the specimen gauge radius may have a great influence on the requested strength of epoxy. As the epoxy and the specimen have same amplitude of torque, the relationship between the required maximum stress of epoxy and the specimen size parameters can be obtained through the torque equal condition as

$$
\tau_{\max }=\frac{4 \bar{r}^{2} R_{b} \delta}{R_{b}^{4}-r_{s}^{4}} \tau
$$

Set the radius of the bar is $12.5 \mathrm{~mm}$ and the thickness of the specimen gauge section is $1 \mathrm{~mm}$, the ratio of epoxy maximum stress to specimen yield stress can be getting as shown in figure 13. In the case of the specimen inner radius is $5 \mathrm{~mm}$, the ratio of the required maximum strength of the epoxy to the specimen material yield stress is only 6.36 percent. However, when the specimen inner radius increases to $7.5 \mathrm{~mm}$, this ratio increases to 15.06 percent. This indicates that much higher epoxy strength is needed for the larger inner diameter specimen. Sometimes, it is difficult to find an epoxy with satisfied strength, especially when the tested material has very high yield strength.

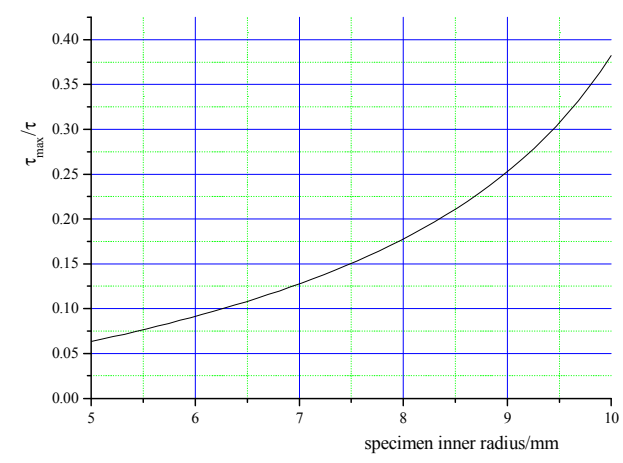

Fig.13. The variety of ratio between epoxy maximum stress to specimen yield stress with the specimen inner radius.

\section{Conclusion}

Numerical simulations on torsional SHB experiments were carried out with LS-DYNA. The deformation characteristics of the specimen during loading process were studied, and the influence of specimen size and other factors on the experimental results was analysed.

The agreement between numerically derived strainstress results and the specimen material properties specified in numerical modelling indicates that the torsional SHB is applicable to study the dynamic shear behaviour of materials under high strain rates. The stress of the specimen has good uniformity in the loading process, while the plastic strain of the material is proportional to the distance from the axis.

With the increase of specimen thickness, the amplitude of corresponding transmission wave signal also increases in proportion. And the increase of specimen thickness will directly lead to the increase of the stress and strain non-uniformity of the specimen. A specimen gauge thickness of no more than $1 \mathrm{~mm}$ is suggested.

Numerical simulation shows that the increase of the inner diameter of the specimen has no effect on the experimental results. Still, much higher epoxy strength is needed for the larger inner diameter specimen. A relationship between the required maximum stress of epoxy and the specimen parameters is given, and the design of specimen gauge diameter should be based on the material yield stress level and the strength of epoxy.

Financial support from National Natural Science Foundation of China (Grant number 11572299 and 11472257) for carrying out this study is acknowledged.

\section{References}

1. W.E. Baker, C.Y. Yew. J. Appl. Mech. 33 (1966)

2. T. Nicholas, J.E. Lawson. J. Mech. Phys. Solids, 20 (1972)

3. A. Gilat, C.S. Cheng. Exp. Mech. 40,1 (2000)

4. A. Khosravifard, M.M. Moshksar, R. Ebrahimi. Mat. Design. 52 (2013)

5. B.J. Claus, X. Nie, B.E. Martin, W. Chen. Exp. Mech, 55 (2015)

6. L.H. Dai, Y.L. Bai, S.W.R. Lee. Comp. Sci. Tech. 58 (1998)

7. W.R. Whittington, A.L. Oppedal, S. Turnage, Y. Hammi, H. Rhee, P.G. Allison, C.K. Crane, M.F. Horstemeyer. Mat. Sci. Eng. A, 594 (2014)

8. F. Yazdani.a, M.N. Bassim., A.G. Odeshi. Proc. Eng. (2009)

9. D.A. Shockey, J.W. Simons, C.S. Brown, T. Kobayashi. Exp. Mech. 47 (2007)

10. T. Kobayashi, J.W. Simons, C.S. Brown, D.A. Shockey. Int. J. Impact Eng. 35 (2008)

11. N.K. Naik, A. Asmelash, V.R. Kavala, Ch. Veerraju, Mech. Mat.39 (2007)

12. A.G. Odeshi, G.M. Owolabi, M.N.K. Singh, and M.N. Bassim, Metal. Mat. Trans. A, 38A, 11 (2007) 